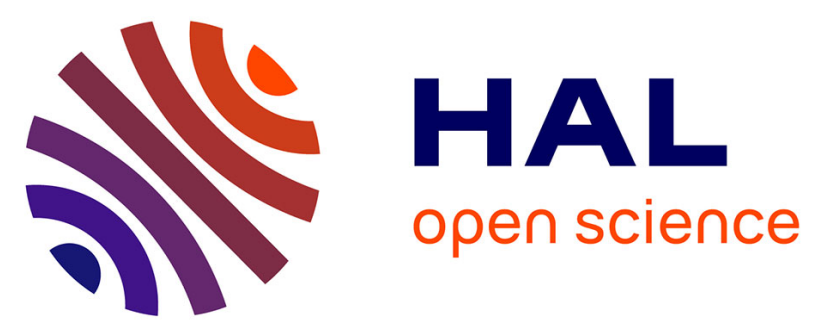

\title{
Magnetic Stochastic Oscillators: Noise-Induced Synchronization to Underthreshold Excitation and Comprehensive Compact Model
}

Alice Mizrahi, Nicolas Locatelli, Rie Matsumoto, Akio Fukushima, Hitoshi Kubota, Shinji Yuasa, Vincent Cros, Joo-Von Kim, Julie Grollier, Damien S Querlioz

\section{To cite this version:}

Alice Mizrahi, Nicolas Locatelli, Rie Matsumoto, Akio Fukushima, Hitoshi Kubota, et al.. Magnetic Stochastic Oscillators: Noise-Induced Synchronization to Underthreshold Excitation and Comprehensive Compact Model. IEEE Transactions on Magnetics, 2015, 51 (11), pp.1 - 4. 10.1109/TMAG.2015.2439736 . hal-01826839

\section{HAL Id: hal-01826839 \\ https://hal.science/hal-01826839}

Submitted on 7 Jul 2018

HAL is a multi-disciplinary open access archive for the deposit and dissemination of scientific research documents, whether they are published or not. The documents may come from teaching and research institutions in France or abroad, or from public or private research centers.
L'archive ouverte pluridisciplinaire HAL, est destinée au dépôt et à la diffusion de documents scientifiques de niveau recherche, publiés ou non, émanant des établissements d'enseignement et de recherche français ou étrangers, des laboratoires publics ou privés. 


\title{
Magnetic stochastic oscillators: Noise-induced synchronization to under-threshold excitation and comprehensive compact model
}

\author{
Alice Mizrahi ${ }^{1,2}$, Nicolas Locatelli ${ }^{1}$, Rie Matsumoto ${ }^{3}$, Akio Fukushima ${ }^{3}$, \\ Hitoshi Kubota ${ }^{3}$, Shinji Yuasa ${ }^{3}$,Vincent Cros $^{2}$, Joo-Von Kim ${ }^{1}$, Julie Grollier ${ }^{2}$, and Damien Querlioz ${ }^{1}$ \\ ${ }^{1}$ Institut d'Electronique Fondamentale, CNRS UMR 8622, Université Paris Sud, 91405 Orsay, France \\ 2 Unité Mixte de Physique CNRS/Thales, 91767 Palaiseau, and Université Paris Sud, 91405 Orsay, France \\ ${ }^{3}$ National Institute of Advanced Industrial Science and Technology (AIST), Tsukuba, Japan
}

\begin{abstract}
Superparamagnetic tunnel junctions are noise powered stochastic oscillators, which can harness thermal energy through phenomena such as stochastic resonance and noise-induced synchronization. This enables them to operate with minimal externally supplied energy and therefore makes them promising candidates for implementing bioinspired computing schemes. These applications require understanding how superparamagnetic tunnel junctions can be integrated into CMOS circuits. In this work, we present a compact model of superparamagnetic tunnel junction, written in the VerilogA language, that can be used within standard integrated circuit design tools. This compact model is based on the Néel-Brown model and allows for fast simulations. We show that this model can reproduce the experimental characterization of a sample subjected to different values of DC currents. Then we definitively demonstrate the validity of the model by confronting it to experimental results in the case of a complex phenomenon: noise-induced synchronization.
\end{abstract}

Index Terms-Spintronics, bioinspired computing, stochastic resonance, magnetic tunnel junctions.

\section{INTRODUCTION}

$\mathbf{M}$ AGNETIC tunnel junctions are considered to be a breakthrough technology for non volatile memories as the cell of magnetic random access memories (MRAM). However, they can also be engineered to be stochastic oscillators powered by noise, in which case they are called superparamagnetic tunnel junction (SMTJs) [1]-[4]. It has been shown that these junctions can exhibit stochastic resonance [2]-[4], a phenomenon where noise enables a non-linear system to detect or synchronize on weak signals [5], [6]. Neuroscience research suggests that our brain takes advantage of stochastic resonance to perform more uncertainty tolerant and less power consuming computations [7], [8]. In addition to their ability to harness noise, the small size and CMOS compatibility of magnetic tunnel junctions makes them promising candidates to implement novel computing schemes [9]. By harnessing thermal energy, bioinspired computing applications based on SMTJs would tackle the issue of increasing power consumption and poor resilience to noise in processors. However, using noise-induced phenomena in SMTJs for bioinspired application requires a better understanding of the behavior of SMTJs and in particular of how they can be integrated in hybrid SMTJ/CMOS circuits.

Because of the utility of MTJs for memory applications [10], [11], there is a lot of interest in developping so-called "compact models" of MTJs that can be used along with CMOS within standard integrated circuit design tools such as the Cadence platform [12]-[15]. Nevertheless, these models consider traditional MTJs in their switching regime and not SMTJs. Therefore, in this work, we propose a compact model of superparamagnetic tunnel junction and demonstrate its validity by comparing simulations to experimental results.

Corresponding author: A. Mizrahi (email: alice.mizrahi@u-psud.fr).
This paper is organized as follows: we introduce SMTJs and their behavior as noise-powered stochastic oscillators, we present our compact model and compare it with a simple experimental characterization of a SMTJ, and finally we confront our model with experimental results in the case of a complex phenomenon: noise-induced synchronization.

\section{Superparamagnetic MTJS A NOISE-POWERED OSCILLATORS}

MTJs are composed of two magnetic layers separated by a tunnel barrier. The magnetization of the top magnetic layer (free layer) has two stable states: parallel or anti-parallel to the magnetization of the lower magnetic layer (reference layer), which is pinned (Fig. 1). Parallel magnetizations (P state) lead to a low electric resistance $\left(R_{P}\right)$ whereas anti-parallel magnetizations (AP state) lead to a high electric resistance $\left(R_{A P}\right)$. MTJs are mainly used for data storage [10], [11] and are therefore usually designed to have a high energy barrier separating the $\mathrm{P}$ and AP state. On the contrary, SMTJs are engineered to have a low energy barrier, so that the thermal energy at room temperature is high enough to induce switching of the magnetization between the P and AP states (Fig. 1(b)). SMTJs thus behave as stochastic oscillators (Fig. 1(c)) which operate with thermal noise and do not require any external supply of energy [1]. The SMTJ is characterized by its dwell times, which are the time intervals spent in the AP and $\mathrm{P}$ states (Fig. 1(c)). We work with the assumption that the free layer can be seen as a single domain magnetization element, therefore its magnetization reversal can be considered within the Kramer's transition rates theory [16]. The magnetization has a probability to switch from the state AP $(\mathrm{P})$ to the state $\mathrm{P}$ (AP) between time 0 and time $t$ :

$$
P_{A P / P}(t)=1-\exp \left(-\frac{t}{<\tau_{A P / P}>}\right)
$$



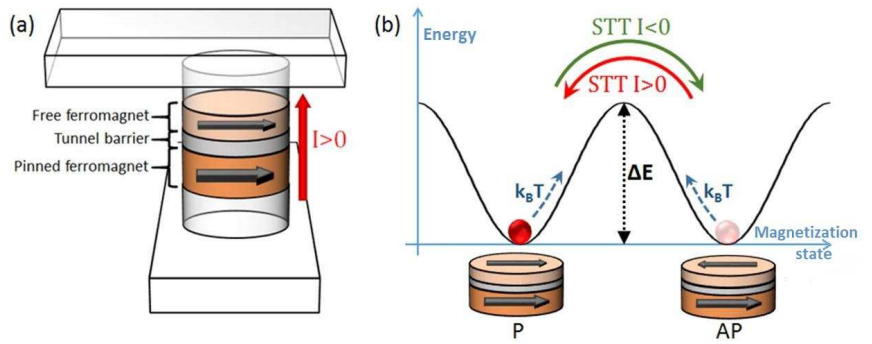

(c)

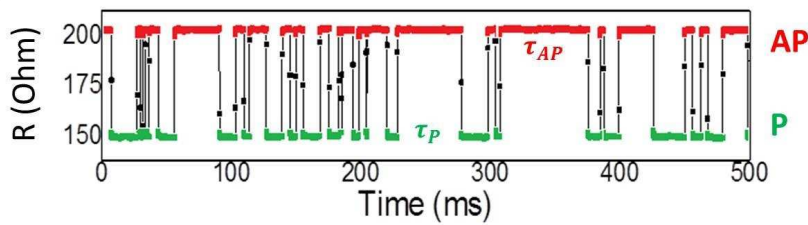

Fig. 1. (a) Schematics of a MTJ. (b) Spin transfer torque and thermal switching in a SMTJ: the energy of the system is plotted versus the magnetization state of the free layer. (c) Experiments: Resistance of a SMTJ as a function of time, featuring the dwell-times in the Anti-Parallel (red) and Parallel (green) states. The asymmetry between the P and AP states is due to the spin transfer torque effect generated by a small DC current into the junction.

as described by the Néel-Brown model [17], [18]. Thus the dwell times follow a Poisson process of characteristic time $\left\langle\tau_{A P / P}>\right.$ which corresponds to the mean dwell time in the AP (P) state. Therefore, even though the SMTJ does not oscillate with a constant period we can define its mean number of oscillations by second - or natural frequency:

$$
F=\frac{1}{<\tau_{A P}>+<\tau_{P}>} .
$$

The mean dwell times are given by the Arrhenius equations: $<\tau_{A P / P}>=\tau_{0} \exp \left(\frac{\Delta E}{k_{B} T}\right)$ where $\tau_{0}$ is the effective attempt time, $\Delta E$ is the energy barrier between the $\mathrm{AP}$ and $\mathrm{P}$ states, $k_{B}$ is the Boltzmann constant and $T$ is the temperature. The stability of both states can be influenced magnetically and electrically. The application of an external magnetic field parallel (anti-parallel) to the magnetization of the reference layer (i.e. the easy axis of the junction) stabilizes the parallel (antiparallel) state by modifying the energy landscape. Through spin transfer torque (STT) effect, a positive (negative) current injected in the SMTJ destabilizes the parallel (anti-parallel) state [19]-[21] - as illustrated on Fig. 1(a-b) - which can be described as a modification of the effective temperature [1], [18]:

$$
<\tau_{A P / P}>=\tau_{0} \exp \left(\frac{\Delta E}{k T}\left(1 \pm \frac{V}{V_{c}}\right)\left(1 \mp \frac{H-H_{0}}{H_{k}}\right)^{n}\right),
$$

where $V$ is the voltage across the junction, $V_{c}$ is the threshold switching voltage, $H$ is the external magnetic field applied along the easy axis of the junction, $H_{k}$ is the anisotropy and $n$ is a real number exponent. $H_{0}$ is the residual stray field from the reference layer. Optimal fabrication of the junction with a perfectly balanced synthetic antiferromagnet (SAF) would lead to a situation where $H_{0}=0$, but in practice $H_{0}$ can reach several Oersteds.

The SMTJ used for the experimental results in this work are elliptical pillars of $60 \times 180 \mathrm{~nm}^{2}$ composed of a SAF trilayer of $\mathrm{CoFe}(2.5 \mathrm{~nm}) / \mathrm{Ru}(0.85 \mathrm{~nm}) / \mathrm{CoFeB}(3 \mathrm{~nm})$ as reference layer, an $\mathrm{MgO}$ tunnel barrier $(1.05 \mathrm{~nm})$, and a CoFeTiB $(2 \mathrm{~nm})$ free layer (Fig. 1(a)). Twelve samples were studied, here we present we results for two of them.

\section{COMPREHENSIVE COMPACT MODEL FOR FAST SIMULATIONS AND SAMPLE CHARACTERIZATION}

It is important to have models of SMTJs that are compatible with the tools used by both academic and industrial researchers to design integrated circuits including CMOS technology. In consequence, we propose a compact model written in the VerilogA language, that can be used within standard design tools, such as the Cadence Spectre simulator. In order to perform fast simulations, this model is not based on the full magnetic Landau-Lifshitz-Gilbert equation but on the NéelBrown model presented in section II. At each step the program computes the mean dwell-times $\left\langle\tau_{A P}\right\rangle$ and $\left\langle\tau_{P}\right\rangle$ and the corresponding probability to switch from the current state to the other, according to equations (1) and (3). A random number is generated to take the decision to switch or not. The model does not take into account the dynamics of the magnetization inside each energy well (intra-well dynamics) and hence does not take switching duration into account. Therefore the model is valid if the dwell-times are large compared to the time scale of the intra-well dynamics, which will be the case in this paper because $<\tau_{A P / P}>$ is larger than $10^{-5}$ s whereas intrawell dynamics have time constant in the order of nanoseconds [12]. In order to have time efficient simulations and not lose the advantage of the compact model, it is important that the time step is not too small. On the other hand, to accurately reproduce the Néel-Brown model, the time step must be small compared to the dwell times. We use the VerilogA command boundstep which allows to set a variable upper limit on the time step. A satisfying compromise is to compute boundstep as a hundredth of the current mean dwell time at each step. The model allows the user to set all the parameters present in equation (3), as well as the resistance of the parallel and anti-parallel states. The influence of the field like torque can also be taken into account by modifying the effective magnetic field in equation (3) [1] but is neglected in this paper.

We test our model by comparing it with the experimental characterization of a SMTJ. In sample $\mathrm{n} 1, R_{P} \simeq 130 \Omega$ and $R_{A P} \simeq 165 \Omega$. The measurements are conducted at room temperature. Fig. 2(a) represents the mean dwell times for the parallel and anti-parallel states as functions of the injected DC current, for various external magnetic fields, at room temperature. Fig. 2(b) illustrates the magnetic field dependency of the SMTJ. The injected current for which the probability to be in each sate $\mathrm{AP}$ or $\mathrm{P}$ is $50 \%\left(<\tau_{A P}>=<\tau_{P}>\right)$ is plotted as a function the applied magnetic field. For both cases, we observe that the simulations results (solid line) match the experimental results (symbols). Three parameters are extracted from the experimental data then finely tuned to obtain this match: $\Delta E / k T=11.3, V_{c}=0.18 \mathrm{~V}$ and $H_{k}=57$ Oe. We set $\tau_{0}=1 \mathrm{~ns}$ [1] and $n=2$ which are the appropriate values for a single domain particle with uniaxial anisotropy [1], [22]. These results suggest the validity of the Néel-Brown 


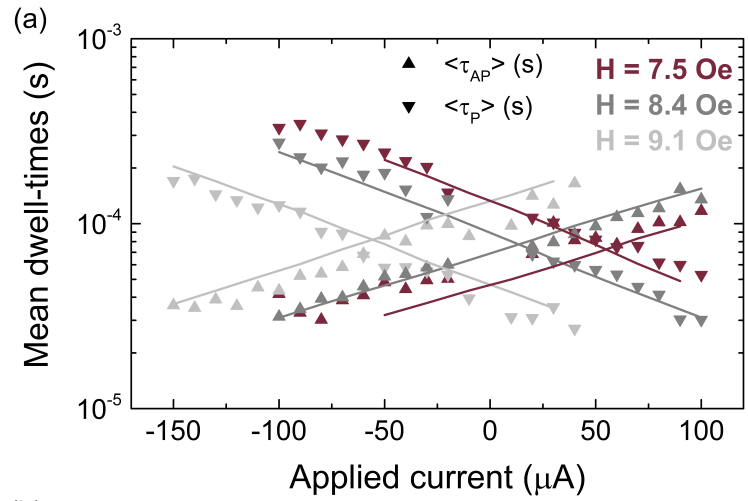

(b)

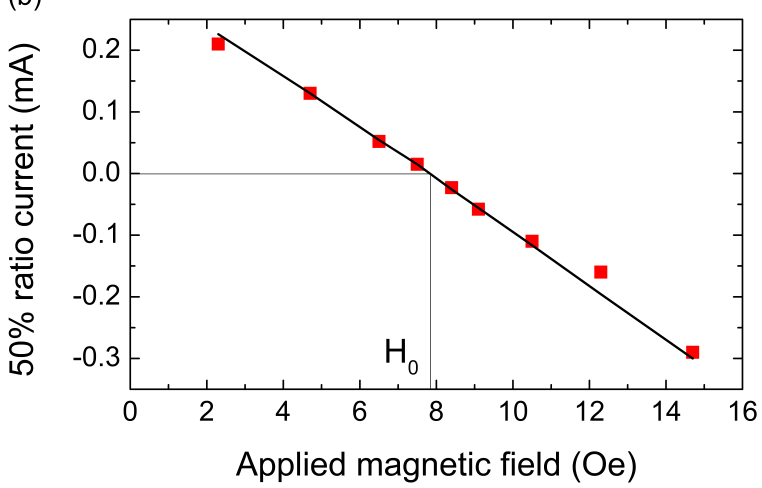

Fig. 2. Experiments on sample n1. (a) Mean dwell times for the antiparallel (up triangle) and parallel (down triangle) states as functions of the applied current for various applied magnetic fields. (b) Current for which $\left\langle\tau_{A P}\right\rangle=\left\langle\tau_{P}\right\rangle$ as a function of the applied magnetic field. $H_{0}$ is the necessary field to compensate the residual field of the SAF. For both graphs experimental measurements (symbol) are compared with simulations based on the compact model (lines).

model with spin transfer torque to describe SMTJs as well as its implementation in our compact model.

Fig. 2(b) enables us to extract the value $H_{0}=7.8$ Oe for the sample presented here. As this is usually not optimal for applications, we experimentally apply an external magnetic field along the easy axis of the junction in order to compensate $H_{0}$.

\section{COnFrontation of THE COMPACT MODEL With A COMPLEX PHENOMENON: NOISE-INDUCED SYNCHRONIZATION}

Here, we want to prove the validity of our compact model for the understanding of more complex phenomena, with view of exploring bioinspired computing applications. Therefore, we confront our model to experimental results that exhibit highly non linear behavior: stochastic resonance and noiseinduced synchronization of a SMTJ [2], [3]. Stochastic resonance has been observed in spin valves [23], [24] and more recently in MTJs [4] as a mean to detect a weak AC signal. In Ref. [2] we demonstrated that stochastic resonance can be used to synchronize a SMTJ to a weak periodic current signal. Here, we reproduce these experimental results with Cadence Spectre simulations based on our compact model. Experiments are conducted with the sample $\mathrm{n} 2$, a SMTJ for which $R_{P} \simeq 150 \Omega$ and $R_{A P} \simeq 200 \Omega$. At room temperature, a square periodic current is applied to the SMTJ, with a subthreshold amplitude. This means that the STT effect itself is not sufficient to trigger the switching, but instead modulates the probability for the magnetization to switch. The switches are thus only allowed by the presence of thermal noise and are thus of stochastic nature. We monitor the resistance of the SMTJ for different current amplitudes and frequencies in order to perform a time resolved analysis of its response.

We use two criteria to quantify synchronization, both represented on Fig. 3. The first criterion is the synchronization rate (Fig. 3(a)), which corresponds to the proportion of time spent in the same state (up/AP or down/P) by both the excitation and the oscillator. The second criterion is the excitation influence on the frequency of the stochastic oscillator (Fig. 3(b)). For both criteria, we observe that simulations results (lines) match experimental results (symbols). The following values are used the simulations: $n=2, \tau_{0}=1 \mathrm{~ns}, \Delta E / k T=16.2$ and $V_{c}=0.25 \mathrm{~V}$. The values for $\Delta E / k T$ and $V_{c}$ were extracted from prior experimental data then finely tuned.

At high input frequencies the excitation is too fast to be followed by the oscillator. Therefore the synchronization rate is low (Fig. 3(a)) and the oscillator frequency does not vary with the excitation frequency and only depends on the current amplitude (Fig. 3(b)). As the input frequency decreases, the system is increasingly able to follow the excitation. In consequence, the matching time increases (Fig. 3(a)) and the oscillator frequency is pulled toward the excitation frequency (Fig. 3(b)). When the excitation frequency is very small compared to the natural frequency of the oscillator at the considered amplitude, supplementary switches (glitches) appear [2]. These are very short oscillations of the SMTJ, leading the oscillator frequency to rise above the excitation frequency (Fig. 3(b)). Because glitches are very short, they do not significantly affect the synchronization rate, which keeps increasing (Fig. 3(a)). These behaviors are the signature of stochastic resonance [2], [25]-[27].

Higher current amplitudes allow wider frequency ranges of synchronization (as the natural frequency of the oscillator is increased) and higher synchronization rate. We observe here that noise-induced synchronization is achieved even at amplitudes as low as $I_{a c}=100 \mu \mathrm{A}$, less than a tenth of the required current to obtain deterministic switching $I_{c}=V_{c} * R \simeq 1.3 \mathrm{~mA}$. Noise-induced synchronization of superparamagnetic tunnel junctions is hence promising for low power computing applications involving synchronization.

\section{CONCLUSION}

We have proposed a compact model for superparamagnetic tunnel junctions and shown its validity by reproducing experimental results in the case of a complex phenomenon. Moreover, the model allows predicting the behavior of SMTJs with different parameters and therefore will be useful for designing optimal SMTJs for future applications. For instance smaller energy barriers - which can be achieved at smaller junction dimensions - lead to higher synchronization frequencies, while lower $V_{c}$ allows achieving synchronization 
(a)

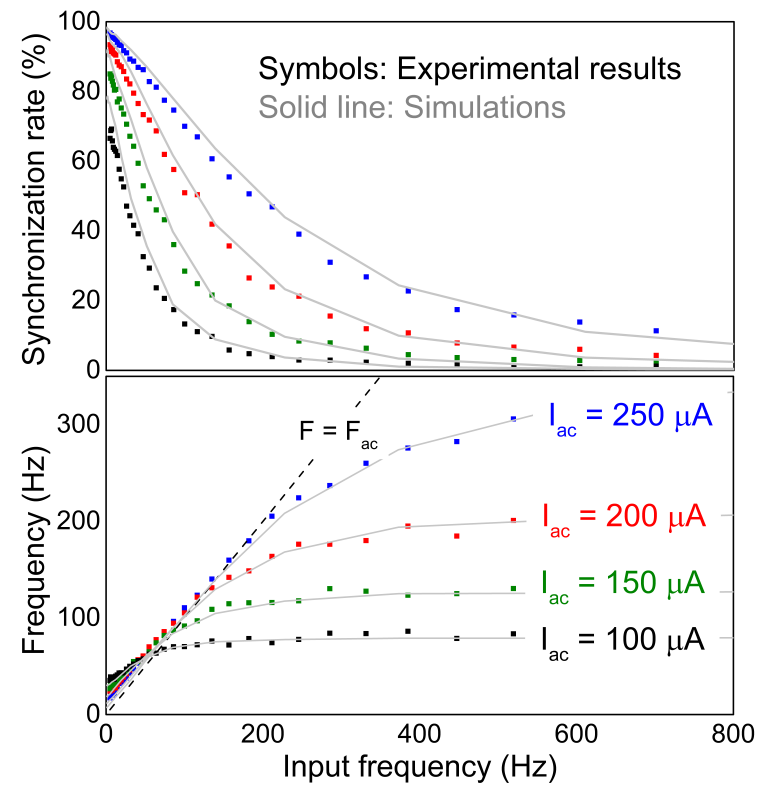

Fig. 3. Experiments on sample n2. (a) Measurements of the synchronization rate of the SMTJ with subthreshold inputs, as a function of input frequency. (b) Mean response frequency of the SMTJ as a function of input frequency. For both graphs, experimental measurements (symbols) are compared with simulations based on the compact model (lines). On the dashed line, the frequency $F$ of the stochastic oscillator is equal to the frequency $F_{a c}$ of the excitation.

at even lower power consumption. This model allows for fast simulations: the totality of the results presented in this paper can be computed in one hour on a standard Intel Xeon CPU. Therefore, the model will enable exploring bioinspired applications involving CMOS and several SMTJs, such as associative memories based on arrays of synchronized SMTJs [28].

\section{ACKNOWLEDGMENT}

The authors acknowledge financial support from the FETOPEN Bambi project No. 618024. A. M. acknowledges financial support from the Ile-de-France regional government through the DIM nano-K program.

\section{REFERENCES}

[1] W. Rippard, R. Heindl, M. Pufall, S. Russek, and A. Kos, "Thermal relaxation rates of magnetic nanoparticles in the presence of magnetic fields and spin-transfer effects," Physical Review B, vol. 84, no. 6, p. 064439, Aug. 2011.

[2] N. Locatelli, A. Mizrahi, A. Accioly, R. Matsumoto, A. Fukushima, H. Kubota, S. Yuasa, V. Cros, L. G. Pereira, D. Querlioz, J.-V. Kim, and J. Grollier, "Noise-Enhanced Synchronization of Stochastic Magnetic Oscillators," Physical Review Applied, vol. 2, no. 3, p. 034009, Sep. 2014.

[3] N. Locatelli, A. Vicent, A. Mizrahi, J. S. Friedman, D. Vodenicarevic, J.V. Kim, J.-O. Klein, W. Zhao, J. Grollier, and D. Querlioz, "Spintronics devices as key elements for energy-efficient neuroinspired architectures," Design, Automation and Test in Europe, 2015.

[4] X. Cheng, C. T. Boone, J. Zhu, and I. N. Krivorotov, "Nonadiabatic Stochastic Resonance of a Nanomagnet Excited by Spin Torque," Physical Review Letters, vol. 105, no. 4, p. 047202, Jul. 2010.

[5] L. Gammaitoni, P. Hänggi, P. Jung, and F. Marchesoni, "Stochastic resonance," Reviews of Modern Physics, vol. 70, no. 1, pp. 223-287, Jan. 1998
[6] S. Bahar, A. Neiman, L. A. Wilkens, and F. Moss, "Phase synchronization and stochastic resonance effects in the crayfish caudal photoreceptor," Physical Review E, vol. 65, no. 5, p. 050901, May 2002.

[7] A. Samardak, A. Nogaret, N. B. Janson, A. G. Balanov, I. Farrer, and D. A. Ritchie, "Noise-Controlled Signal Transmission in a Multithread Semiconductor Neuron," Physical Review Letters, vol. 102, no. 22, p. 226802, Jun. 2009

[8] T. Mori and S. Kai, "Noise-Induced Entrainment and Stochastic Resonance in Human Brain Waves," Physical Review Letters, vol. 88, no. 21, p. 218101, May 2002.

[9] N. Locatelli, V. Cros, and J. Grollier, "Spin-torque building blocks," Nature Materials, vol. 13, no. 1, pp. 11-20, Jan. 2014.

[10] C. Chappert, A. Fert, and F. N. Van Dau, "The emergence of spin electronics in data storage," Nature Materials, vol. 6, no. 11, pp. 813823, Nov. 2007

[11] T. Kawahara, K. Ito, R. Takemura, and H. Ohno, "Spin-transfer torque RAM technology: Review and prospect," Microelectronics Reliability, vol. 52, no. 4, pp. 613-627, Apr. 2012.

[12] A. Vincent, N. Locatelli, J.-O. Klein, W. Zhao, S. Galdin-Retailleau, and D. Querlioz, "Analytical Macrospin Modeling of the Stochastic Switching Time of Spin-Transfer Torque Devices," IEEE Transactions on Electron Devices, vol. 62, no. 1, pp. 164-170, Jan. 2015.

[13] Y. Zhang, W. Zhao, Y. Lakys, J.-O. Klein, J.-V. Kim, D. Ravelosona, and C. Chappert, "Compact Modeling of Perpendicular-Anisotropy $\mathrm{CoFeB} / \mathrm{MgO}$ Magnetic Tunnel Junctions," IEEE Transactions on Electron Devices, vol. 59, no. 3, pp. 819-826, Mar. 2012.

[14] Y. Zhang, W. Zhao, G. Prenat, T. Devolder, J.-O. Klein, C. Chappert, B. Dieny, and D. Ravelosona, "Electrical Modeling of Stochastic Spin Transfer Torque Writing in Magnetic Tunnel Junctions for Memory and Logic Applications," IEEE Transactions on Magnetics, vol. 49, no. 7, pp. 4375-4378, Jul. 2013.

[15] G. Panagopoulos, C. Augustine, and K. Roy, "Physics-Based SPICECompatible Compact Model for Simulating Hybrid MTJ/CMOS Circuits," IEEE Transactions on Electron Devices, vol. 60, no. 9, pp. 28082814, Sep. 2013.

[16] H. A. Kramers, "Brownian motion in a field of force and the diffusion model of chemical reactions," Physica, vol. 7, no. 4, pp. 284-304, 1940

[17] W. F. Brown, "Thermal Fluctuations of a Single-Domain Particle," Physical Review, vol. 130, no. 5, pp. 1677-1686, Jun. 1963.

[18] Z. Li and S. Zhang, "Thermally assisted magnetization reversal in the presence of a spin-transfer torque," Physical Review B, vol. 69, no. 13, p. 134416, Apr. 2004.

[19] J. Slonczewski, "Current-driven excitation of magnetic multilayers," Journal of Magnetism and Magnetic Materials, vol. 159, no. 1-2, pp. L1-L7, Jun. 1996.

[20] J. A. Katine, F. J. Albert, R. A. Buhrman, E. B. Myers, and D. C. Ralph, "Current-Driven Magnetization Reversal and Spin-Wave Excitations in Co/Cu/Co Pillars," Physical Review Letters, vol. 84, no. 14, pp. 3149_ 3152, Apr. 2000.

[21] J. C. Slonczewski, "Currents, torques, and polarization factors in magnetic tunnel junctions," Physical Review B, vol. 71, no. 2, p. 024411, Jan. 2005.

[22] R. H. Victora, "Predicted time dependence of the switching field for magnetic materials," Physical Review Letters, vol. 63, no. 4, pp. 457460, Jul. 1989.

[23] G. Finocchio, I. N. Krivorotov, X. Cheng, L. Torres, and B. Azzerboni, "Micromagnetic understanding of stochastic resonance driven by spintransfer-torque," Physical Review B, vol. 83, no. 13, p. 134402, Apr. 2011.

[24] M. d'Aquino, C. Serpico, R. Bonin, G. Bertotti, and I. D. Mayergoyz, "Stochastic resonance in noise-induced transitions between selfoscillations and equilibria in spin-valve nanomagnets," Physical Review B, vol. 84, no. 21, p. 214415, Dec. 2011.

[25] A. Neiman, A. Silchenko, V. Anishchenko, and L. Schimansky-Geier, "Stochastic resonance: Noise-enhanced phase coherence," Physical Review E, vol. 58, no. 6, pp. 7118-7125, Dec. 1998.

[26] B. Shulgin, A. Neiman, and V. Anishchenko, "Mean Switching Frequency Locking in Stochastic Bistable Systems Driven by a Periodic Force," Phys. Rev. Letters, vol. 75, no. 23, pp. 4157-4160, Dec. 1995.

[27] J. A. Freund, L. Schimansky-Geier, and P. Hänggi, "Frequency and phase synchronization in stochastic systems," Chaos: An Interdisciplinary Journal of Nonlinear Science, vol. 13, no. 1, pp. 225-238, Mar. 2003.

[28] G. Csaba, M. Pufall, D. Nikonov, G. Bourianoff, A. Horvath, T. Roska, and W. Porod, "Spin torque oscillator models for applications in associative memories," in 2012 13th International Workshop on Cellular Nanoscale Networks and Their Applications (CNNA), Aug. 2012, pp. $1-2$. 